\title{
Algorithm for Surface Geometry Identification by Controlled Measuring Clouds of Points
}

\author{
Gabriel Frumuşanu
}

\begin{abstract}
This paper deals with the manner on which a cloud of points should be generated when measuring a part surface and with the processing procedures needing to be applied in order to characterize the geometry of addressed surface. More precise, an algorithm for surface geometry identification has been developed and implemented by MatLab applications. It finds the necessary number of measuring points, according to surface precision requirements, and their distribution, conform to surface functional role. Basic geometrical features of the surface are assessed by using the coordinates of the resulted cloud of points. A numerical simulation has been performed for sampling the algorithm application.
\end{abstract}

Index Terms-Cloud of points, controlled measuring, identification algorithm, surface geometry features.

\section{INTRODUCTION}

During recent years, most of the manufacturing equipments became numerically controlled and suffered outstanding improvements. Congruent to this, the measurement techniques also changed, the parts measuring process being performed by numerical controlled measuring equipments (e.g. coordinates measuring machines CMM-s).

Each coordinate measuring system is based on sampling some points on the surface to inspect [1], the result of such a measurement consisting in a point cloud given through points' coordinates. The measured part feature(s) result subsequently by various operations (partition, extraction, filtration, association, collection and construction) applied to these points and their coordinates.

In manufacturing, dimensional inspection purpose is the part conformity evaluation, this meaning to assess the real part deviation relative to its nominal model. The model of a real surface is fitted on the points from the cloud and the geometric deviation results as the maximum distance between the real and the nominal surface's models. For example, a robust linear programming formulation-based algorithm for straightness and flatness evaluation is introduced in [2]. One should notice that, because only few points usually define the amplitude of the tolerance zone, these points are effectively relevant to evaluate the geometric deviation.

In what concerns the dimensional inspection process, this has become more and more complicated. It requires solutions specific to every different part, regarding the inspection

Manuscript received January 31, 2017; revised March 23, 2017.

G. Frumuşanu is with the Manufacturing Engineering Department, Dunărea de Jos University of Galaţi, Romania (e-mail: gabriel.frumusanu@ ugal.ro). sequence of the part features, the number of measuring points and their locations, the probing paths, and the avoidance of collision between the involved elements. The enhancement of this process' performance, materialized in diminishing the needed time and cost, has been addressed in many researches (e.g. see [3]-[7]). As one can easily suppose, the computer-aided inspection (CAI) has emerged as one of the major topics of the recent research from computer-integrated manufacturing (CIM) [8], [9].

This paper presents a newly developed algorithm for surface geometry identification, implemented by MatLab applications. It finds the necessary number of measuring points, according to surface precision requirements, and their distribution, conform to surface functional role. Basic geometrical features of the surface are assessed by using the coordinates of the resulted cloud of points. The next section gives details of the approach underlying the identification algorithm, which is then presented in the third section. The fourth section is dedicated to a numerical simulation, while the last one is for conclusion.

\section{Problem Formulation}

The manner of performing a dimensional inspection process on CMM and specific procedures for processing the coordinates of the points from the cloud resulted by measurement in order to deliver the required information about the targeted surface geometry are stated in a new generation of ISO / ANSI standards, released after 2010. Despite both vastness and complexity of their content, they still remain aspects to be added / clarified / improved inside the addressed area, some of them being specified below.

- A correlation between the uncertainty of measuring process result, on one hand, and required accuracy of the inspected surface, on the other hand is necessary.

- The dimensional inspection process planning should take into account the inspected surface functional purpose.

- In many cases, the assessment of surface characteristic features, as they are defined in standards, requires an extended volume of (sometimes-complicated) calculus.

By starting from these observations, the new algorithm for surface geometry identification, here presented, has been developed. It supposes integration between the measuring process and the measured points coordinates processing and the following issues characterize it:

- The measuring points' number is chosen after the required accuracy of the inspected surface.

- The measuring points' distribution is modified if the inspected surface is part of a fit. 
- The inspected surface's geometry is primarily identified by assessing three characteristic features: offset, inclination and shape deviation.

\section{IDENTIFICATION ALGORITHM}

The nominal, designed geometry of a part consists in a reunion of surfaces with perfect shape (plane, cylindrical, spherical etc.) and precise dimensions. After manufacturing, the real part results different, its conformity being decided by dimensional inspection. Although it is applicable to any surface shape, for a better understanding, the geometry identification algorithm is further detailed in the case of the real surface corresponding to a plane nominal surface. Hereby, the inspected surface will be referred to a plane, horizontal surface (Fig. 1). In other cases, a similar discussion can be done after changing the model (shape) of the reference surface. The identification algorithm includes the below presented modules: A1, A2 (if necessary) and B.

\section{A. Measuring Points Selection}

\section{1) Measuring points number and surface fitting}

The process of establishing the appropriate number of measuring points, according to the accuracy assigned to the inspected surface, has an iterative character. More precise, the number of measuring points $n$ is successively increased until a control parameter $i_{c}$ reaches a threshold value $i_{p}$, set after prescribed accuracy, according to the flowchart from Fig. 2.

As already mentioned, the points' coordinates are measured on a CMM. A plane can be fitted to these points by using least squares method. If choosing MatLab soft for this purpose, then sftool package finds out plane equation as:

$$
z=f(x, y)=p_{00}+p_{10} \cdot x+p_{01} \cdot y .
$$

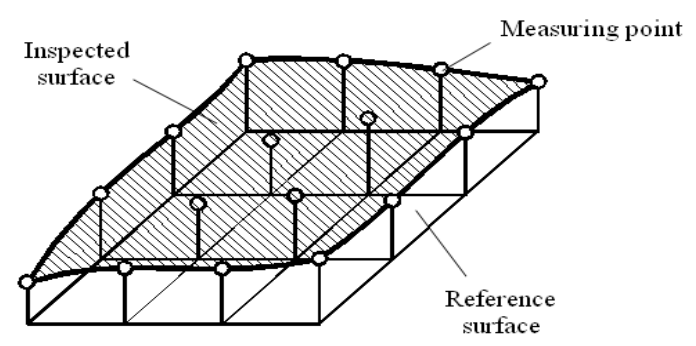

Fig. 1. Inspected versus reference surface.

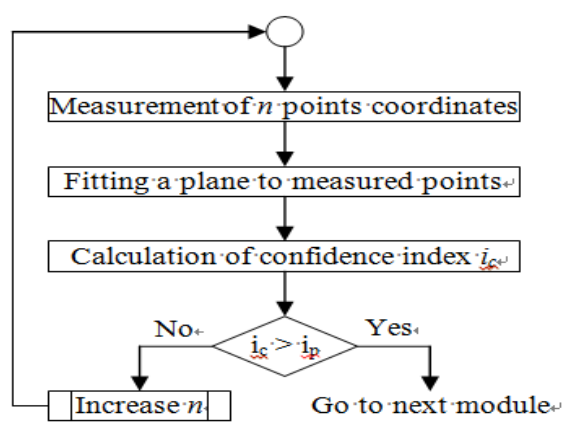

Fig. 2. Establishment of measuring points' number.

In (1), $x$ and $y$ mean the coordinates axis defining the reference plane. Besides fitting plane equation, sftool also delivers some indicators reflecting goodness of fit, among which root-mean-square error (RMSE):

$$
R M S E=\left(\sum_{k=1}^{n}\left(\hat{z}_{k}-z_{k}\right)^{2} / n\right)^{1 / 2}
$$

where $\hat{z}_{k}$ means here the value of $z$ coordinate calculated in $\left(x_{k}, y_{k}\right)$ point with (1), while $z_{k}$ is the measured value in the same point. A RMSE value closer to zero indicates a more accurate fit. By making a trade off between the number of measuring points and the accuracy in fitting a plane to them, we define the confidence index $i_{c}$ as:

$$
i_{c}=n /(1000 \cdot R M S E)
$$

Hereby, the bigger is $i_{c}$ value, the higher is the confidence in measurement result. A number of threshold values $i_{p}$ can be adopted depending on the prescribed accuracy for the inspected surface. For sample, three confidence levels might be considered:

$$
i_{c}<1, i_{c} \in[1,10] \text { and } i_{c}>10 .
$$

Regarding the network of measuring points, at this stage, it is as uniform as possible. The first four measuring points should be placed in the corners of the largest rectangle that can be inserted on the reference surface (Fig. 3-a), its dimensions $L$ and $l$ respecting the conditions:

$$
L=j \cdot l_{s}, l=i \cdot l_{s}, i, j \in N^{*} \text {. }
$$

The number of measuring points is gradually increased until reaching the targeted level of confidence, by following a predefined procedure, illustrated in Fig. 3. The first two multiplications are done in order to mesh the reference surface in squares of $l_{s}$ side (Fig. 3-b, c). The squares are then minced in new squares with halved side after a cycle of three multiplications of the measuring points (Fig. 3-d, e, f). This cycle repeats until the number of measuring points becomes sufficient.

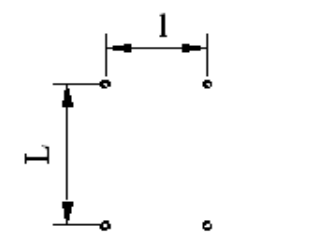

(a)

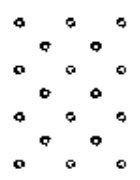

(d)

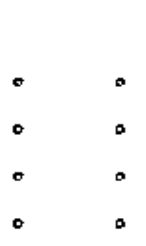

(b)

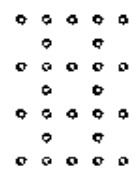

(e)

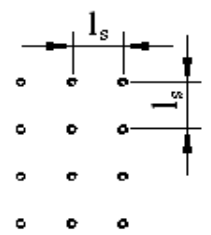

(c)
Fig. 3. Measuring points' multiplication.

The procedure for measuring points' multiplication has been conceived such as the speed of points' number relative 
increase varies as little as possible and, at the same time, remains at reasonable values.

The result of this stage consists in the coordinates of the points cloud corresponding to the last mesh of the reference surface and in the equation of the plane fitted to them.

2) Measuring points distribution

If the inspected surface must not meet any special requirements issuing from its functional role (is not part of a surfaces fit), then after executing the first module of the geometry identification algorithm, one may jump directly to the assessment of surface geometry feature (next module). Otherwise, the points placed above the fitted plane (1) become more important than the other ones, because they are effectively characterizing the nature of the fit formed with the conjugated surface. Hereby, a uniform distribution of the measuring points is no longer suitable.

The manner of taking into account the increased relevance of the higher points from the inspected surface materializes in adding new measuring points around the highest ones (the peaks) from the cloud previously determined. Because in this way the number of measuring points might substantially increase, only a fraction from the highest peaks (e.g. 1/3 or 1/4) will be considered for generating new measuring points.

Regarding new measuring points position relative to the peaks of the inspected surface this is presented in Fig. 4. There are three possibilities: the peak is in the interior of the mesh (Fig. 4-a, b, c), in a corner (Fig. 4-d, e, f) or on one side (Fig. 4-g, h, i).

One should notice that, depending on the stage of the measuring points' multiplication when the confidence level was reached (see subsection A-1), the mesh corresponding to the points cloud in its final structure may have three different aspects (as depicted in Fig. 3. d, e or f).

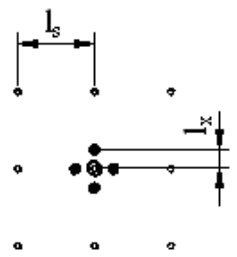

(a)

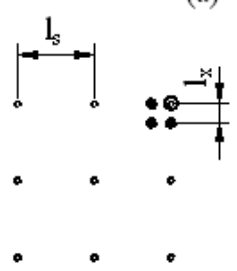

(d)

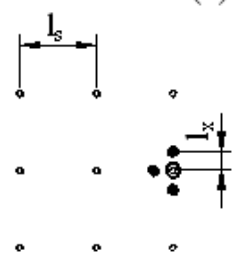

(g)

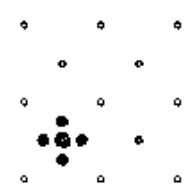

(b)

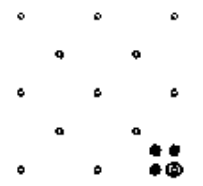

(e)

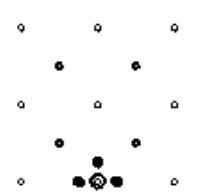

(h)

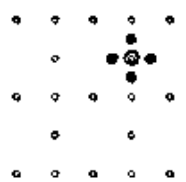

(c)

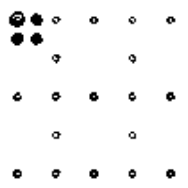

(f)

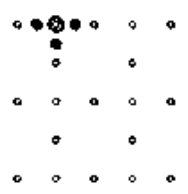

(i)
Fig. 4. Position of measuring points added to the initial cloud.

The magnitude of $l_{x}$ parameter, giving the distance between the peak and the new added measuring points is obviously smaller than squares side $l_{s}$, e.g. $l_{x}=l_{s} / 4$.

\section{B. Assessment of Surface Geometry Features}

Three features, namely offset, inclination and shape deviation [10], can primarily define inspected surface's geometry, as below explained.

- Offset means the average distance between the points from the cloud representing the inspected surface and the reference surface. It is denoted by $o$.

- Inclination respect the axis of an orthogonal reference system $x y$ conveniently adopted in the reference surface means the slope of the line fit to the points obtained by projecting the cloud's points on a plane perpendicular to reference surface and including one of the two axis ( $x z$ or $y z$, Fig. 5). It is denoted by $p_{x}$, respective $p_{y}$.

- Shape deviation $s_{d}$ is the amplitude's maximum value from the Fourier transform applied to the series formed by $z$-coordinate values of the points obtained by projecting the cloud's points on a plane normal to the reference surface (Fig. 6), after taking away both offset and inclination.

Regarding features assessment, the offset is easy to calculate, e.g. in MatLab with mean instruction applied to $z$-coordinate values for all the points of the measured cloud.

In what concerns the inclination evaluation, we refer to the case of inclination relative to $x$-axis, while the other case (inclination relative to $y$-axis) can be addressed similarly.

- $\quad$ The cloud points are projected to $x z$ plane (Fig. 5).

- The resulted points are set in order after the ascending values of $x$ coordinate, which form the vector $I_{x}$. Another vector, $v_{x}$ is formed by $z$-coordinate values of projection points, according to their order in $I_{x}$ vector.

- The cftool package from MatLab deliver the slope $p_{x}$ of the straight line $d_{x}$ fitted by regression to projection points, using the instruction:

$$
p_{x}=\operatorname{polyfit}\left(I_{x}, v_{x}, 1\right) \text {. }
$$

In order to find the shape deviation, from the points of the measured cloud are subtracted offset and inclination determined as above. In this way results a new cloud of points $P(x, y, z)$, which are projected into a plane normal to $x y$, passing by the origin and making a variable angle $\alpha_{0}$ (between 0 and $\pi / 2$ ) with $x$-axis, Fig. 6 . For each projection point, the distance $d$ between its vertical and the origin is calculated:

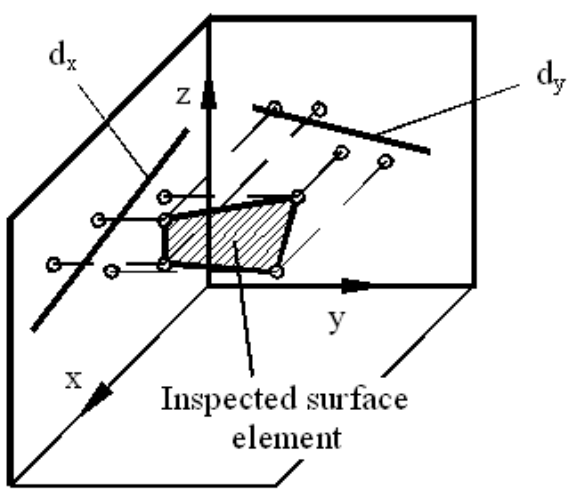

Fig. 5. Inspected surface inclination. 


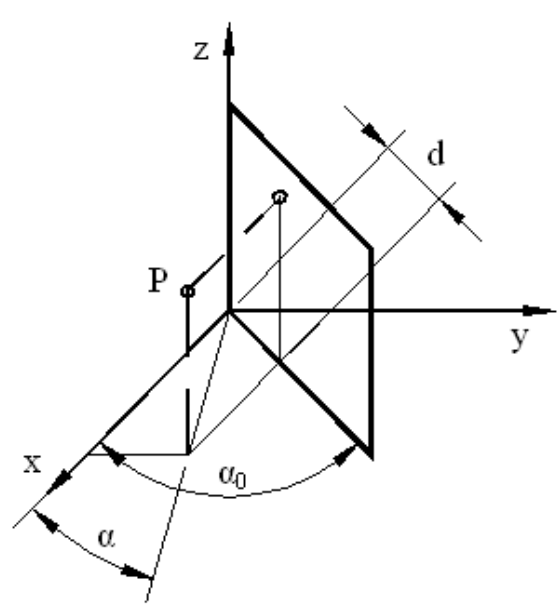

Fig. 6. Shape deviation assessment.

$$
d=\left(x^{2}+y^{2}\right)^{1 / 2} \cdot \cos \left[\alpha_{0}-\arctan (y / x)\right]
$$

A series is formed by stringing after $d$ increasing values the corresponding $z$-coordinate values. A discrete Fourier transform is applied to resulted series - fft command, the amplitude's maximum value being recorded. The maximum value of the maximum amplitude, when $\alpha_{0}$ is varying as above-mentioned, means the shape deviation $s_{d}$ of the corresponding surface. We should notice that $s_{d}$ feature can be calculated only if the number of points from the measured cloud is high enough.

\section{NUMERICAL SimUlATION}

We further present a numerical simulation developed in order to prove the feasibility of the above presented algorithm for surface geometry identification. First, a "real" surface having as reference a horizontal plane (a rectangle) will be generated as a cloud of points. The rectangle is covered by an uniform squared grid, its generic point position being defined by a couple $(i, j)$, the variables $i$ and $j$ meaning the number of the line (parallel to $x$-axis) and of the column (parallel to $y$-axis), respectively. The conversion between $(i, j)$ and $(x, y)$ is immediate, the grid side length being of $0.1 \mathrm{~mm}$. The z-coordinate of the point on the "real" surface corresponding to the generic point, $z_{i j}$ has been adopted as:

$$
\begin{aligned}
z_{i j} & =z_{0}+(i-1) p_{i}+(j-1) p_{j}+A_{i} \sin \left[(i-1) \phi_{i}\right]+ \\
& +A_{j} \cos \left[(j-1) \phi_{j}\right]+\varepsilon_{i j}, \quad i=1 \quad 101, j .=1 \quad 141, \ldots
\end{aligned}
$$

where $z_{0}$ means an offset-type deviation, in the origin of the coordinates system $(i=1, j=1)$. Second and third terms give to surface an inclination relative to $x$ and $y$-axis respectively, proportional to the values of $p_{i}$ and $p_{j}$ parameters. Fourth and fifth term give a composed waviness of the surface, while the last, $\varepsilon_{i j}$, is a random variable simulating the surface roughness.

Dedicated MatLab applications have been developed in order to perform each module of the identification algorithm. The first one successively multiplies the number of measuring points $n$, according to the procedure illustrated in
Fig. 3 (starting from 4 points in corners) and in every case assesses the corresponding confidence index $i_{c}$. If the input values in (8) are $h_{0}=0.12 \mathrm{~mm}, p_{i}=-0.001 \mathrm{~mm}, p_{j}=-0.0005$ $\mathrm{mm}$,

$A_{i}=0.02 \mathrm{~mm}, A_{j}=0.01 \mathrm{~mm}, \varphi_{i}=\pi / 10 \mathrm{rad}$ and $\varphi_{i}=\pi / 10 \mathrm{rad}$, then the confidence index values depending on the number of measuring points are presented in Table I and Fig. 7.

Another application has been conceived for implementing the second module of the identification algorithm. It detects the highest points from the inspected surface and multiplies the number of measuring points around these peaks, according to the procedure presented in Fig. 4. There are two levels of multiplication. Let us suppose that the cloud of points measured during module A1 has 12 points (Fig. 8-a). At the first level, the highest 3 points (1/4 from 12) are selected and new measuring points are added (Fig. 8-b). At the second level, the new highest 6 points (1/4 from 24) are retained and new measuring points are added (Fig. 8-c).

TABLE I: THE CONFIDENCE INDEX VALUES

\begin{tabular}{cccccccccc}
\hline \hline $\mathrm{n}$ & 4 & 8 & 12 & 18 & 26 & 35 & 59 & 87 & 117 \\
\hline $\mathrm{i}_{\mathrm{c}}$ & 1.05 & 1.29 & 2.07 & 2.56 & 4.02 & 5.22 & 4.62 & 7.39 & 9.85 \\
\hline \hline
\end{tabular}

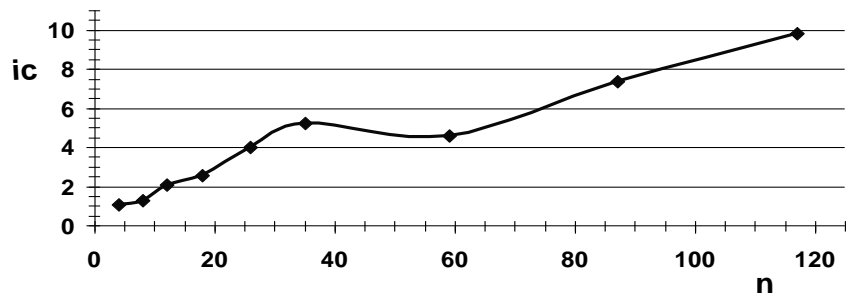

Fig. 7. Confidence index versus measuring points number.

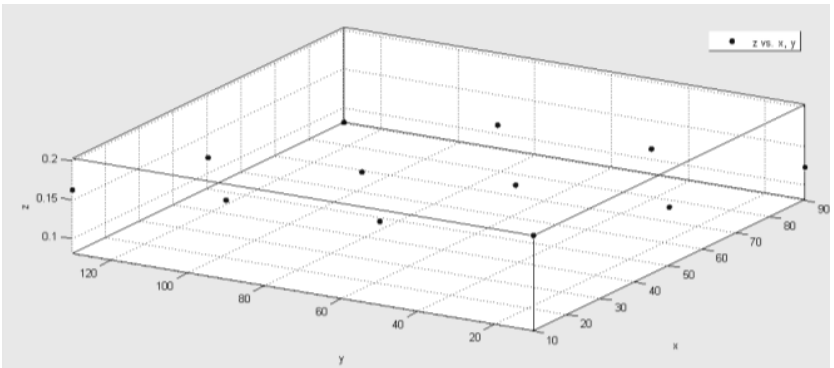

(a)

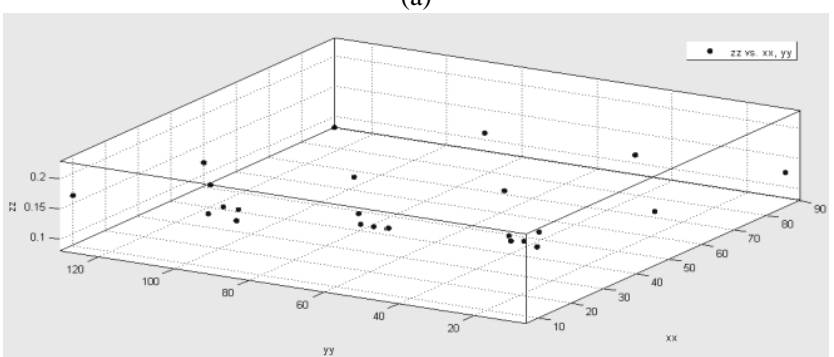

(b)

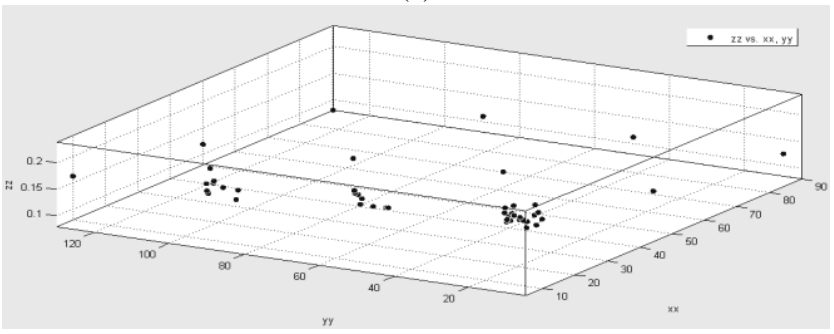

(c)

Fig. 8. Multiplication of measuring points near the inspected surface peaks 
A third application has been designed in order to assess the geometry features values for the simulated surface, according to the procedures presented when introducing the third module of the identification algorithm (section III B). The results obtained by successively running this application in four cases are presented in Table II. First time the measured cloud included 12 points $\left(i_{c}=2.07\right)$, then 35 points $\left(i_{c}=5.22\right)$, 117 points $\left(i_{c}=9.85\right)$ and finally 14241 points (the maximum possible number $=101 \times 141$, hence $i_{c}$ also has the maximum possible value).

TABLE II: THE VALUES OF ASSESSED FEATURES

\begin{tabular}{cccc}
\hline \hline $\mathrm{i}_{\mathrm{c}}$ & $\mathrm{o}[\mathrm{mm}]$ & $\mathrm{p}_{\mathrm{x}}[\mathrm{mm}]$ & $\mathrm{p}_{\mathrm{y}}[\mathrm{mm}]$ \\
\hline 2.07 & 0.1465 & -0.0093 & -0.0035 \\
\hline 5.22 & 0.1447 & -0.0106 & -0.0043 \\
\hline 9.85 & 0.1368 & -0.0103 & -0.0047 \\
\hline $\max$ & 0.1343 & -0.0109 & -0.0051 \\
\hline \hline
\end{tabular}

The shape deviation value has been determined only for the maximum number of points, resulting $s_{d}=0.0145 \mathrm{~mm}$.

The offset values calculated in the three cases presented in Fig. 8 are $o_{1}=0.1465 \mathrm{~mm}$ ("uniform" cloud formed by 12 points), $o_{2}=0.1683 \mathrm{~mm}$ (after the first multiplication of measuring points) respective $o_{3}=0.1885 \mathrm{~mm}$ (after the second multiplication).

If analyzing the numerical results of the simulation, the following remarks arise:

- The confidence index is consistently defined and it can be a very useful criterion in establishing the measuring points' number relative to the required accuracy of the inspected surface. We estimate that $i_{c}=5$ should correspond to ISO precision class 7 .

- The suggested modification of measuring points' distribution when the inspected surface forms a fit with a conjugated surface is effective. Offset value increase after multiplying the measuring points around inspected surface's peaks gives more realistic information about the clearance / allowance in the considered fit.

- $\quad$ The proposed features quickly offer basic information about the inspected surface's geometry and the methodology for assessing these features is effective.

- The assessed values of the features characterizing the inspected surface's geometry are convergent to the real values when the measurement confidence index increases (see Table II).

\section{CONCLUSION}

This paper presents a new algorithm for surface geometry identification. It can be used for online controlling the dimensional inspection of the machined surfaces on CMM. By imposing a threshold value for the confidence index (a newly defined indicator), depending on the required accuracy of the inspected surface, the number of measuring points is automatically found. If the inspected surface is part of a fit, an optimized distribution of the measuring points is adopted aiming to obtain a better evaluation of the fit nature. The features suggested to assess the inspected surface geometry image are intuitive and easy to use in establishing the conformity to required geometry. The proposed algorithm has been tested on diverse simulated geometries of inspected surface and the obtained results are encouraging for extending its future application.

\section{REFERENCES}

[1] G. Moroni and S. Petro, "Optimal inspection strategy planning for geometrical tolerance verification,” Precision Engineering, vol. 38, pp. 71-81, 2014.

[2] S. Cho and J.-Y. Kim, "Straightness and flatness evaluation using data envelopment analysis," Int J Adv Manuf Technol, vol. 63, pp. 731-740, 2012.

[3] S. H. Mian and A. Al-Ahmari, "Enhance performance of inspection process on coordinate measuring machine," Measurement, vol. 47, pp. 78-91, 2014.

[4] G. Moroni and S. Petro, "Inspection strategies and multiple geometric tolerances," Procedia CIRP, vol. 10, pp. 54-60, 2013.

[5] B. C. Jiang and S.-D. Chiu, "Form tolerance-based measurement points determination with CMM," Journal of Intelligent Manufacturing, vol. 13, pp. 101-108, 2002.

[6] H. Zhao, J.-P. Kruth, N. Van Gestel, B. Boeckmans, and P. Bleys, "Automated dimensional inspection planning using the combination of laser scanner and tactile probe," Measurement, vol. 45, pp. 1057-1066, 2012.

[7] H.-C. Chang and A. C. Lin, "An innovative algorithm for static sampling of measured points and simplifying measuring probe orientation for sculpture surfaces," Int J Adv Manuf Technol, vol. 41, pp. 780-798, 2009.

[8] M.-W. Cho, H. Lee, G.-S. Yoon, and J. Choi, "A feature-based inspection planning system for coordinate measuring machine," Int $J$ Adv Manuf Technol, vol. 26, pp. 1078-1087, 2005.

[9] M.-W. Cho, H. Lee, G.-S. Yoon, and J. Choi, "A computer-aided inspection planning system for on-machine measurement - Part II: local inspection planning," KSME International Journal, vol. 18, pp. 1358-1367, 2004.

[10] G. Frumuşanu and A. Epureanu, "Part accuracy management by topological mapping of deviations," Applied Mechanics and Materials, vol. 859 , pp. 210-216, 2017.

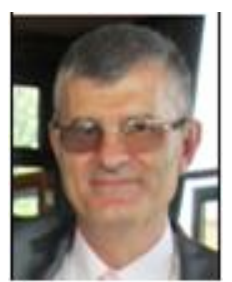

Gabriel Frumuşanu was born in Galaţi, Romania on Sept. 28, 1964. He got the bachelor degree in 1988 got Ph.D in 1999. He published habilitation in 2016 in Industrial Engineering at "Dunărea de Jos" University of Galaţi, Romania.

He is currently a professor at "Dunărea de Jos" University of Galaţi, Romania, in the Manufacturing Engineering Department. He published over 150 scientific articles, some of them in prestigious journals (The Internatinal Journal of Advanced Manufacturing Technology, Indian Journal of Engineering and Material Sciences, International Journal of Mechanics, Materials and Manufacturing). He owns 3 patents. He participated at numerous International conferences (Spain, Hungary, Tunisia, Israel, Moldova and Romania). Research interests in machining systems control, cutting tools profiling and environmental impact of the manufacturing process.

Prof. Frumuşanu is member of UASTRO, of editorial boards - Journal of Control and Systems Engineering (JCSE, Bowen Publishing), Proceedings in Manufacturing Systems journal (Romanian Academy), The Annals of "Dunărea de Jos" University, Fascicle V and is scientific reviewer for prestigious ISI Journals (Proceedings of the Institution of Mechanical Engineers, Part B: Journal of Engineering Manufacture, Proceedings of the Institution of Mechanical Engineers, Part C: Journal of Mechanical Engineering Science, Journal of Mechanical Science and Technology). 